\title{
Fluorescence and interactions with thiol compounds of Nile Red-adsorbed gold nanoparticles
}

\author{
Kun-Hong Lee ${ }^{\text {a }}$, Shih-Ju Chen ${ }^{\text {a }}$, Jing-Yueh Jeng ${ }^{\text {b }}$, Yu-Ching Cheng ${ }^{\text {b }}$, Jen-Taie Shiea ${ }^{\text {b }}$, \\ Huan-Tsung Chang a,c,* \\ a Department of Chemistry, National Taiwan University, Roosevelt Road, Sec. 4, Taipei 106, Taiwan \\ ${ }^{\mathrm{b}}$ Department of Chemistry, National Sun Yat-Sen University, Kaohsiung, Taiwan \\ ${ }^{c}$ Department of Natural Science Education, National TaiTung University, TaiTung, Taiwan
}

Received 7 September 2006; accepted 3 December 2006

Available online 9 December 2006

\begin{abstract}
We have investigated the interactions between a variety of thiols and Nile Red-adsorbed gold nanoparticles (NRAuNPs). After adding thiols to solutions of NRAuNPs, the solutions fluoresce strongly as a result of the displacement of a Nile Red-derived product from the surface of the AuNPs. We propose a mechanism for the formation of this NR product on the surface of AuNPs by conducting mass spectrometry, fluorescence, and capillary electrophoresis measurements. By recording the fluorescence changes of the NRAuNP solutions after addition of the thiols, we investigated the interactions between the thiols and NRAuNPs. Using the Langmuir isotherm model, we found that the displacement rate constants for thiols having one carboxyl residue, such as 3-mercaptopropionic acid, fall within the range $0.55-1.19 \times 10^{-2} \mathrm{~s}^{-1}$. Thiols containing hydroxyl groups [e.g., 2-mercaptoethanol (2-ME)] or amino groups [e.g., $N$-(2-mercaptopropionyl)glycine (MPG)], or that have flat structures on the AuNP surface, such as mercaptosuccinic acid, exhibit double-exponential kinetics with first rate constants of $0.51-2.83 \times 10^{-2} \mathrm{~s}^{-1}$ and second rate constants of 6.0-23.4 $\times 10^{-4} \mathrm{~s}^{-1}$. Our results reveal that steric effects and the charge density of the thiols both play important roles in determining the interactions with NRAuNPs. The interactions (displacement and/or induced aggregation) are also dependent on the size of NRAuNPs.
\end{abstract}

(c) 2006 Elsevier Inc. All rights reserved.

Keywords: Adsorption; Fluorescence; Gold nanoparticle; Kinetic; Nile Red; Thiol

\section{Introduction}

A great deal of effort has been directed toward elucidating the surface coverage, structure, function, and dynamics of immobilized thiols on electrodes and metallic surfaces, primarily because of their crucial implications for the design of novel functionalized materials [1-3]. Many thiol-immobilized gold nanoparticles (AuNPs) that are stable in aqueous solution are used to prepare protein-, carbohydrate-, and DNAfunctionalized AuNPs for the optical sensing of biomolecules such as proteins and DNA [1,4-6]. The three most important factors that affect the magnitude of the optical changes are (1) the extent of surface modification of the gold probes, (2) the

\footnotetext{
* Corresponding author. Fax: +886233661171.

E-mail address: changht@ @ntu.edu.tw (H.-T. Chang).
}

ability of the analytes to gain access to the surface-bound molecules, and (3) the ability of the surface-bound molecules to recognize the analytes. Understanding these factors is essential for controlling the sensitivity of the gold probes.

A number of techniques, including ${ }^{1} \mathrm{H}$ NMR spectroscopy, infrared spectroscopy, X-ray photon spectroscopy (XPS), and surface plasmon resonance (SPR) spectroscopy, have been employed to characterize functionalized AuNPs [3,7-10]. Fluorescence spectroscopy, by comparison, is a relatively versatile and sensitive technique for exploring the nature of chemical species on a surface [4,11-14]. Increasingly, fluorescence spectroscopy based on energy transfer is utilized to study the dynamics and thermodynamics of AuNPs [4,11,15-18]. When fluorophorelabeled analytes bind to AuNPs, their fluorescence becomes quenched as a result of energy transfer and collisions between the fluorophore and the AuNPs. Once the fluorophore-labeled analytes become displaced by thiols, such as 2-mercaptoethanol 
(2-ME), they diffuse to the bulk solution and fluoresce strongly. For example, a fluorescence method has been developed to determine both the number of thiol-derivatized single-stranded oligonucleotides bound to AuNPs and the extent of their hybridization with complementary oligonucleotides in solution [5]. Murray and coworkers investigated the effects that the concentration and the chain length of the incoming species have on the kinetics of the place-exchange reaction by conducting fluorescence and ${ }^{1} \mathrm{H}$ NMR spectroscopy measurements and suggested an " $\mathrm{S}_{\mathrm{N}} 2$-type" associative mechanism to explain their experimental results $[18,19]$. These fluorescence studies also suggest that the insertion of the incoming thiol in the preformed lattice weakens the strength of interaction of several adsorbed molecules, which results in the rearrangement of more than a single unit during the reaction rate-determining step.

Fluorescent dyes bound to colloidal particles have been used to sense a number of analytes of interest, including DNA and proteins $[20,21]$. In a previous study, we prepared Nile Red (NR)-bound AuNPs (NRAuNPs) and used them as sensors for thiols; the limits of detection (LOD)_defined as a signal-tonoise ratio $(\mathrm{S} / \mathrm{N})$ of 3 -were in the $\mathrm{nM}$ regime for most of the thiols we tested, including cysteine and homocysteine [4]. Solutions containing the as-prepared NRAuNPs fluoresce weakly as a result of fluorescence resonance energy transfer, electron transfer, and collisions between the NR and the AuNPs, but they fluoresce strongly at $\mathrm{pH} 4.0$ in the presence of thiols because of the displacement of an NR product into the bulk solution.

In this paper, we propose a mechanism for the formation of the NR product and investigated the interactions between thiols and NRAuNPs. By monitoring the fluorescence changes that occurred during the course of the reaction with various thiols, we used the Langmuir isotherm model [22-24] to obtain the rate of displacement of the NR product from the AuNP surface. In addition, we also sought to investigate the effects that the charges of the thiols have on their interactions with different sizes of NRAuNPs.

\section{Experimental}

\subsection{Chemicals}

Sodium tetrachloroaurate(III) dehydrate, cresyl violet and resorufin was obtained from Sigma (St. Louis, MO). Dimethylsulfoxide, meso-2,3-dimercaptosuccinic acid (DMSA), methanol, glutathione (GSH), 2-mercaptoethanol (2-ME), 16-mercaptohexadecanoic acid (MHA), $N$-(2-mercaptopropionyl)glycine (MPG), 3-mercapto-1,2-propanediol (2-MP), 3-mercaptopropionic acid (MPA), mercaptosuccinic acid (MSA), 11-mercaptoundecanoic acid (MUA), sodium hydroxide, and trisodium citrate were purchased from Aldrich (Milwaukee, WI). NR was obtained from Acros (Geel, Belgium). A citrate buffer prepared from citric acid was adjusted with $\mathrm{NaOH}$ to $\mathrm{pH}$ 4.0.

\subsection{Synthesis of 14-, 32-, and 56-nm-diameter AuNPs}

AuNPs were prepared by reduction of sodium tetrachloroaurate with trisodium citrate that also acted as a capping agent to stabilize the as-prepared AuNPs. By carefully controlling the amount of citrate in the synthesis process, different sizes of AuNPs could be synthesized [25,26]. With increasing the citrate concentration, smaller sizes of AuNPs were prepared. Trisodium citrate $[1 \%(\mathrm{w} / \mathrm{v}) ; 0.8,0.5$, or $0.3 \mathrm{~mL}]$ was added rapidly to three respective aliquots of $0.01 \% \mathrm{HAuCl}_{4}$ that were heated under reflux. The mixtures (final volumes: $50 \mathrm{~mL}$ each) were heated under reflux for an additional $8 \mathrm{~min}$, during which time the color changed to deep red, wine red, and pale red, respectively. The solutions were set aside to cool to room temperature; they were stable for at least 6 months. We denote the concentration of the as-prepared AuNPs to be $1.0 \times$.

\subsection{Preparation of $N R A u N P s$}

A stock solution of NR (1.0 mM) was prepared in dimethylsulfoxide and diluted with dimethylsulfoxide when necessary. Three aliquots of $1.0 \mathrm{mM} \mathrm{NR}(5.0 \mu \mathrm{L}$ each) were added separately to $1.0 \times 14-, 32-$, and 56-nm-diameter AuNP solutions $(5.0 \mathrm{~mL}$ each) prepared in citrate solution at $\mathrm{pH} 4.0$. Please note that the concentrations of these AuNP solutions are ca. $3.4 \times 10^{-9}, 2.9 \times 10^{-10}$, and $5.4 \times 10^{-11} \mathrm{M}$, respectively. Although the adsorption of NR onto the AuNPs is very fast (equilibrium time $<10 \mathrm{~min}$ ), the solutions were set at ambient temperature and pressure overnight (at least $10 \mathrm{~h}$ ) because the formation of the NR product requires ca. $8 \mathrm{~h}$ to reach completion. The solutions were then subjected to three repeated cycles of centrifugation at $12,000 \mathrm{rpm}$ (relative centrifugal force: $16,000 \mathrm{~g}$ ) for $10 \mathrm{~min}$ and washing with citrate solution at $\mathrm{pH} 4.0$ $(5.0 \mathrm{~mL})$. Finally, the precipitates were re-suspended in citrate solution $(1.0 \mathrm{mM})$ at $\mathrm{pH} 4.0$.

\subsection{Characterization of AuNPs and NRAuNPs}

A double-beam UV-vis spectrophotometer (Cintra 10e) obtained from GBC (Victoria, Australia) was used to measure the absorbances of the AuNPs and NRAuNPs in citrate solutions. The UV-vis absorption spectra (not shown) display maximum wavelengths of the SPR bands for the 14-, 32-, 56-nm-diameter AuNPs at 518, 526, and $535 \mathrm{~nm}$, respectively; these values suggest that the sizes of the AuNPs are as expected. The SPR bands for the NRAuNP solutions are close to those for the corresponding AuNP solutions. The sizes and the distributions $( \pm 5.0 \%)$ of the AuNPs and NRAuNPs were further confirmed by transmission electron microscopy (TEM) analysis using a Hitachi H7100 TEM (Tokyo, Japan) operating at $75 \mathrm{kV}$. A fluorometer (Aminco Bowman) obtained from ThermoSpectronic (Pittsford, NY, USA) was used to collect the fluorescence spectra of NR and the NRAuNPs in the presence and absence of thiols. The emission spectra were recorded while separately irradiating the solutions of NR and the NR product at 580 and $480 \mathrm{~nm}$.

\subsection{Measurements of the molecular mass of the NR product}

Prior to measuring molecular masses, the solution of the NRAuNPs was subjected to three repeated cycles of cen- 
trifugation (12,000 rpm for $10 \mathrm{~min})$ and washing with water $(10 \mathrm{~mL})$ to minimize the interference of salt. 2-ME $(10 \mu \mathrm{M}$, $5 \mathrm{~mL}$ ) was added to the precipitate, and the solution was subjected to sonication for $1 \mathrm{~min}$. After $10 \mathrm{~min}$, the solution was centrifuged at $12,000 \mathrm{rpm}$ for $10 \mathrm{~min}$ and then the supernatant was concentrated (ca. 50-fold) using a rotary evaporator. A BioTOF electrospray-ionization time-of-flight mass spectrometer (Bruker Daltonics, Billerica, USA) was used to record mass spectra of the product, which was dissolved in 50\% aqueous methanol solution and infused into a sheathless capillary at a flow rate of $80 \mu \mathrm{L} / \mathrm{h}$.

\subsection{Coverage of NR on AuNPs}

Different concentrations of NR $(0.1-10 \mu \mathrm{M})$ were added separately to the 14-, 32-, and 56-nm-diameter AuNP solutions. From the reflection point of the plot of the fluorescence intensity at $653 \mathrm{~nm}$ vs the concentration of NR (not shown), the saturated NR concentration was estimated and, consequently, the surface coverage of NR was obtained according to the expression $N_{\text {ads }} / 4 \pi r^{2}$, where $N_{\text {ads }}$ is the maximum adsorption number of NR and $r$ is the average radius of the AuNPs [27].

\subsection{Kinetic measurements of NR adsorption}

The fluorescence intensities at $653 \mathrm{~nm}$ of the solutions were recorded immediately after NR was added to the $1.0 \times$ AuNPs under stirring. Unless otherwise noted, the final NR concentration was $1.0 \mu \mathrm{M}$. The data were recorded every $20 \mathrm{~s}$ during the course of a reaction time of $20 \mathrm{~min}$.

\subsection{Kinetic measurements of the displacement rate of the NR products by thiols}

The fluorescence intensities were recorded immediately after various thiol solutions $(2-\mu \mathrm{L}$; final concentrations: $0.1 \mathrm{mM})$ were added separately to the $1.0 \times$ NRAuNPs (final volumes: $2.0 \mathrm{~mL}$ ). To minimize scattering interference, the emission wavelengths were set at $600 \mathrm{~nm}$ for the 14-nm-diameter NRAuNPs and at $610 \mathrm{~nm}$ for the 32- and 56-nm-diameter NRAuNPs. The data were recorded every $20 \mathrm{~s}$ over a period of $60 \mathrm{~min}$.

\subsection{Analysis of kinetic data}

Microcal Origin 6.0 (OriginLab, Norththampton MA, USA) was used to analyze the kinetic data. The kinetic data for NR adsorption were fitted to a second-order exponential decay function; the kinetic data for the displacement of the NR products by thiols were fitted with either a single- or double-exponential monomolecular growth model. The starting parameters- $A_{1}, A_{2}, \tau_{1}, \tau_{2}, K_{1}^{\prime}, K_{2}^{\prime}, k_{\mathrm{obs} 1}$, and $k_{\mathrm{obs} 2}$-were set arbitrarily; $A_{1}$ and $A_{2}$ are preexponential factors, $\tau_{1}$ and $\tau_{2}$ denote the decay times of two different quenching processes, $K_{1}^{\prime}$ and $K_{2}^{\prime}$ are parameters related to $k_{\mathrm{a}}$ and $k_{\mathrm{d}}$ (discussed later), and $k_{\mathrm{obs} 1}$ and $k_{\mathrm{obs} 2}$ are the observed rate constants.

\subsection{FT-IR measurement}

MHA, MPA, MSA and DMSA were added to $1 \times$ NRAuNP solution respectively and the final concentration of thiols was $0.1 \mathrm{mM}$. Let the solution stand for an hour and excess thiols were removed by centrifugation twice at 12,000 rpm for $10 \mathrm{~min}$, followed by decantation of supernatants. Then the pellets were air-dry to obtain the powder samples for FT-IR measurements. The powder was then mixed with $\mathrm{KBr}$ powder and the mixtures were ground into fine powders. The powders were pressed into thin films at 5000 psi. The FT-IR spectra were collected for 16 scans at a resolution of $4 \mathrm{~cm}^{-1}$ from 400 to $4000 \mathrm{~cm}^{-1}$ using a JASCO FT/IR-410 spectrometer.

\section{Results and discussion}

\subsection{Formation of the NR product}

The fluorescence intensity of the NRAuNP solution is very weak, but it is much stronger in the presence of thiols such as 2-ME. In addition, the maximum excitation and emission wavelengths of the NRAuNP solutions in the presence of thiols are 480 and $610 \mathrm{~nm}$, respectively; these values are different from those (580 and $652 \mathrm{~nm}$, respectively) of the NR solution. The product's molecular ion occurs at $m / z$ 282, which is different from that of NR $(m / z 318)$, and it formed a fragment at $\mathrm{m} / z 247.0$ after collision-induced dissociation. The ratio of the intensities of the peaks at $m / z 282.1$ and 284 is 3:1, which suggests the existence of a chlorine atom in the product. On the basis of the MS data, we suggest that the chemical formula for the NR product is $\mathrm{C}_{16} \mathrm{H}_{8} \mathrm{ClNO}_{2}$; i.e., the product was formed by substitution of the $\mathrm{N}\left(\mathrm{C}_{2} \mathrm{H}_{5}\right)_{2}$ residue in NR with a chloride ion from $\mathrm{HAuCl}_{4}$. Scheme 1 presents a possible mechanism for the formation of the product derived from NR on the surface of the AuNPs. It is interesting to note that this product did not form when we added $\mathrm{Cl}^{-}$and 2-ME to a NR solution ( $\mathrm{pH} 4.0)$ in the absence of the AuNPs, nor did we observe when we performed the reaction using silver or silica nanoparticles in place of the AuNPs. It is our belief, therefore, that the AuNPs play a catalytic role in the reaction $[11,28,29]$.

It is known that the large shifts that occur in the observed positions of the absorption and emission bands of NR as a function of solvent polarity are due to large changes in its excited state dipole moment. In polar solvents, this phenomenon results from the near-complete charge separation between the diethylamino and quinoid moieties of the molecule, which may be explained by formation of a twisted intramolecular charge transfer state (TICT) that is due to rotation of the flexible diethylamino group attached to the rigid structure of the molecule [30,31]. Our finding of blue shifts in the absorption and emission bands of the NR product $\left(\mathrm{C}_{16} \mathrm{H}_{8} \mathrm{ClNO}_{2}\right)$ supports this hypothesis. Because of the lack of formation of a TICT in the new product, its quantum yield is greater than that of NR.

Cresyl violet and resorufin exhibit stronger fluorescence than does NR in aqueous solution, as Table 1 indicates. In a man- 


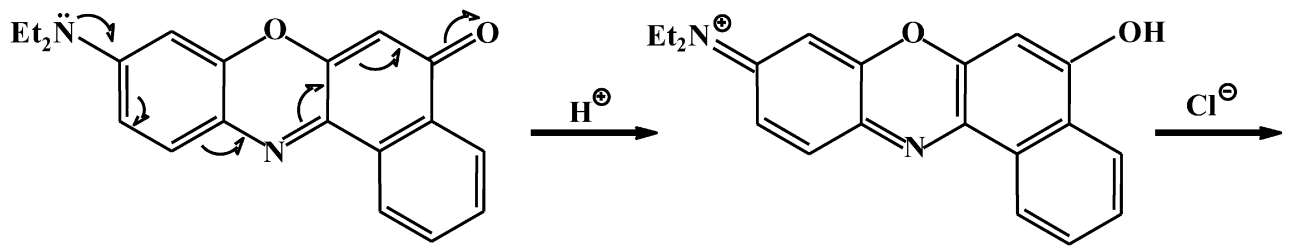

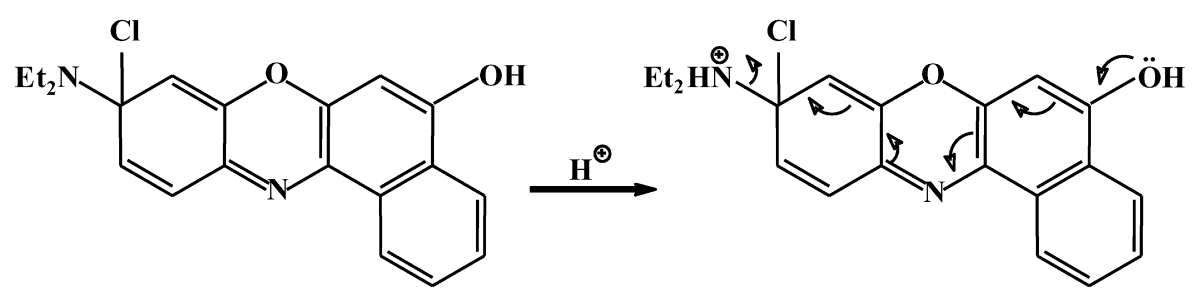

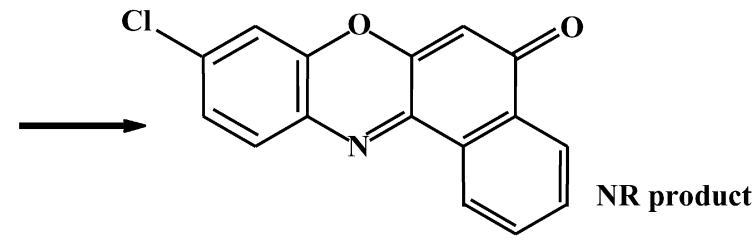

Scheme 1. Mechanism for the formation of the NR product on the AuNPs.

Table 1

Optical properties of three dyes in solutions and of dye-AuNPs in the absence and presence of 2-ME at $\mathrm{pH} 4.0^{\mathrm{a}}$

\begin{tabular}{|c|c|c|c|}
\hline & Nile Red & Cresyl violet & Resorufin \\
\hline$\lambda_{\text {ex }}(\text { dye-AuNP })^{c}$ & 480 & 580 & 560 \\
\hline$\lambda_{\text {em }}(\text { dye-AuNP })^{\mathrm{c}} / \mathrm{FI}$ & $610 / 0.13$ & $622 / 0.11$ & $581 / 0.76$ \\
\hline$\lambda_{\mathrm{em}}(\text { dye-AuNP })^{\mathrm{d}} / \mathrm{FI}$ & $610 / 3.25$ & $621 / 4.92$ & $581 / 1.14$ \\
\hline
\end{tabular}

a The concentrations of NR, cresyl violet, resorufin are all $1 \mu \mathrm{M}$, while those for the 32-nm-diameter AuNPs and 2-ME are $0.3 \mathrm{nM}$ and $0.1 \mathrm{mM}$, respectively.

b Fluorescence intensity (a.u.).

c Equilibration of these dyes with the 32-nm-diameter AuNPs requires at least $8 \mathrm{~h}$.

d Reacted with 2-ME.

ner similar to the quenching effect on NR, the AuNPs also caused significant decreases to occur in the fluorescence intensities of cresyl violet and resorufin. Table 1 indicates, however, that the AuNPs did not exert any catalytic activity for forming new products from cresyl violet and resorufin, even though their structures are similar to that of NR; presumably this situation occurs because the quaternization and leaving group ability both decrease in the order $\mathrm{N}(\mathrm{Et})_{2}>\mathrm{NH}_{2}>\mathrm{OH}$. The fluorescence intensities of the cresyl and resorufin solutions containing AuNPs in the presence of 2-ME were weaker than those in the absence of AuNPs, indicating that the displacements did not reach completion within $10 \mathrm{~min}$. At $\mathrm{pH}$ 4.0, resorufin possesses the greatest hydrophobicity of the three dyes and, therefore, it has the strongest interaction with the AuNPs, which leads to a lower degree of displacement in the presence of 2-ME.

\subsection{Kinetic measurements of $N R$ adsorption and rates of thiol displacement}

Fig. 1 presents the time evolution of fluorescence quenching by the 32-nm-diameter AuNPs; it reaches its minimum value after $10 \mathrm{~min}$. Although the adsorption of NR onto the AuNPs is fast, the rate of product formation is much slower-it requires at least $8 \mathrm{~h}$ to reach equilibrium [4]. The fluorescence follows a multiexponential decay curve, and, thus, we used biexponential decay kinetics (Eq. (1)) to analyze the data:

$F(t)=A_{1} \exp \left(-t / \tau_{1}\right)+A_{2} \exp \left(-t / \tau_{2}\right)$,

where $\tau_{1}$ and $\tau_{2}$ denote the decay times of two different quenching processes and $A_{1}$ and $A_{2}$ are preexponential factors. By taken the assumption that $k_{1}=\left(\tau_{1}\right)^{-1}$ and $k_{2}=\left(\tau_{2}\right)^{-1}$, fitting the fluorescence decay curves with Eq. (1) provides NR 


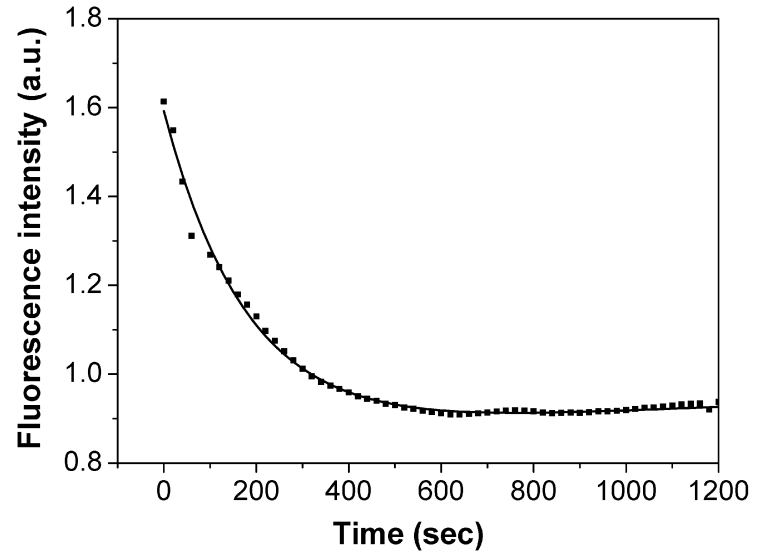

Fig. 1. Fluorescence decay of NR in the presence of 32-nm-diameter AuNPs. Fitting curve (-); experimental data (ם). The fluorescence intensities at $653 \mathrm{~nm}$ were recorded every $20 \mathrm{~s}$. The concentrations of NR and AuNPs were $1 \mu \mathrm{M}$ and $0.3 \mathrm{nM}$, respectively.

Table 2

FT-IR spectroscopic peak assignments for thiols and thiol-adsorbed AuNPs

\begin{tabular}{llllll}
\hline & $\begin{array}{l}v_{\mathrm{a}}^{\mathrm{a}}\left(\mathrm{CH}_{2}\right) \\
\left(\mathrm{cm}^{-1}\right)\end{array}$ & $\begin{array}{l}v_{\mathrm{s}}^{\mathrm{b}}\left(\mathrm{CH}_{2}\right) \\
\left(\mathrm{cm}^{-1}\right)\end{array}$ & $\begin{array}{l}v(\mathrm{SH}) \\
\left(\mathrm{cm}^{-1}\right)\end{array}$ & $\begin{array}{l}v(\mathrm{COOH}) \\
\left(\mathrm{cm}^{-1}\right)\end{array}$ & $\begin{array}{l}v_{\mathrm{s}}\left(\mathrm{CO}_{2}^{-}\right) \\
\left(\mathrm{cm}^{-1}\right)\end{array}$ \\
\hline MHA & $2922^{\mathrm{c}}$ & $2855^{\mathrm{c}}$ & 2559 & 1700 & $1411^{\mathrm{d}}$ \\
MHA-Au & $2920^{\mathrm{d}}$ & $2851^{\mathrm{d}}$ & N.D & 1636 & $1410^{\mathrm{c}}$ \\
MPA & N.D. & N.D. & 2563 & 1709 & $1421^{\mathrm{d}}$ \\
MPA-Au & N.D. & N.D. & N.D. & 1644 & $1396^{\mathrm{c}}$ \\
MSA & N.D. & N.D. & 2548,2565 & 1695 & $1426^{\mathrm{d}}$ \\
MSA-Au & N.D. & N.D. & N.D. & 1643 & $1398^{\mathrm{c}}$ \\
DMSA & N.D. & N.D. & 2537,2562 & 1697 & $1422^{\mathrm{d}}$ \\
DMSA-Au & N.D. & N.D. & N.D. & 1645 & $1397^{\mathrm{c}}$ \\
\hline
\end{tabular}

\footnotetext{
a Asymmetric vibrational peak.

b Symmetric vibrational peak.

c Broad peak.

d Sharp peak.

e Not detected.
}

adsorption rate constants $k_{1}$ and $k_{2}$ of $5.44 \times 10^{-3} \mathrm{~s}^{-1}$ and $8.35 \times 10^{-4} \mathrm{~s}^{-1}$, respectively; we attribute the different rate constants to arise most likely from various adsorption processes $[18,32]$. The faster kinetic step is probably the displacement of the previously adsorbed ions like citrate on the AuNPs [32]. When the surface coverage of NR increases to ca. $80 \%$, the adsorption rate should decrease as a result of steric effects among the NR molecules on the AuNP surface [32-34]. This slower reaction is characterized by the rate constant $k_{2}$.

The fluorescence of the NRAuNP solution increases as a result of desorption of the NR product from the AuNP surface upon the addition of thiols that are involved in strong $\mathrm{Au}-\mathrm{S}$ bonding with the AuNPs. Table 2 lists some of the FTIR spectroscopy data, which indicate the disappearance of the absorption of the SH groups (ca. $2550 \mathrm{~cm}^{-1}$ ) and the shifts of the symmetric and asymmetric stretching bands for the carboxylic acid groups to lower energies in the presence of the NRAuNPs $[35,36]$. These observations support the notion that the thiols bound to the NRAuNPs.

Fig. 2 indicates that the fluorescence at $610 \mathrm{~nm}$ of the solution increases with time after adding 3-mercaptopropionic acid (MPA), and reaches a plateau after $6 \mathrm{~min}$. In this study, we used

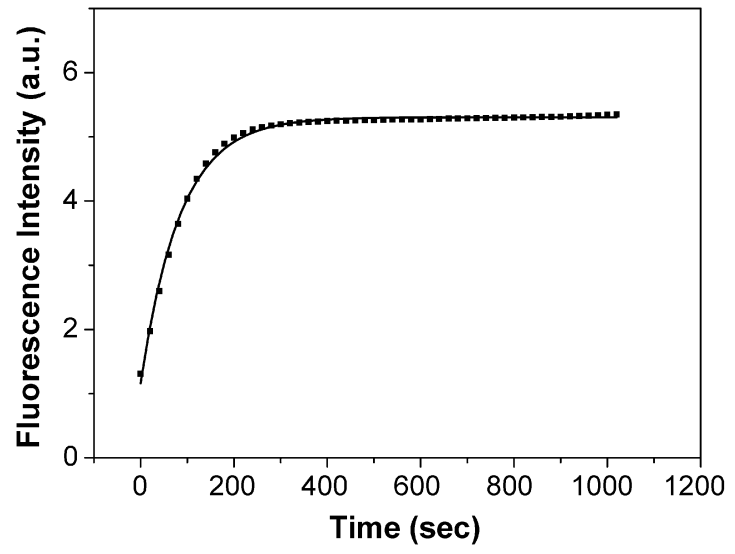

Fig. 2. Fluorescence growth upon replacing the adsorbed NR product molecules on the NRAuNPs with MPA. Fitting curve (-); experimental data (ם). The fluorescence intensities at $610 \mathrm{~nm}$ were recorded every $20 \mathrm{~s}$. The concentrations of the NRAuNPs and MPA were $0.3 \mathrm{nM}$ and $0.1 \mathrm{mM}$, respectively.

thiols at concentrations higher than that of NR so that their diffusion rates from the bulk solution to the interface can be assumed to remain constant from the viewpoint of diffusioncontrolled kinetics. Under such a situation, the kinetics of the process is determined predominantly by the rate of adsorption of the thiols. Therefore, the adsorption rate is first-order in the adsorbing species and first-order in the free sites at the surface. Provided that the thiols adsorbed on the AuNPs are limited to one monolayer, we can use the Langmuir adsorption isotherm (Eq. (2)) to calculate the adsorption rate [22,24,37]:

$\mathrm{d} \theta / \mathrm{d} t=k_{\mathrm{a}}(1-\theta) C-k_{\mathrm{d}} \theta$,

where $\theta$ is the fraction of surface covered, $(1-\theta)$ is the fraction of surface exposed, $C$ is the thiol concentration, and $k_{\mathrm{a}}$ and $k_{\mathrm{d}}$ are the association and dissociation constants, respectively. Integration of Eq. (2) yields the time course for monolayer formation:

$\theta(t)=K^{\prime}\left[1-\exp \left(-k_{\mathrm{obs}} t\right)\right]$

where $K^{\prime}=C /\left[C+\left(k_{\mathrm{d}} / k_{\mathrm{a}}\right)\right]$ and $k_{\mathrm{obs}}=k_{\mathrm{a}} C+k_{\mathrm{d}}$. If we consider that the thiol molecules immediately replace the NR product from the AuNP surface once they are adsorbed, the magnitude of the fluorescence is proportional to the surface coverage of the NR product. Thus Eq. (3) can be further modified into

$F(t)=K^{\prime}\left[1-\exp \left(-k_{\mathrm{obs}} t\right)\right]$

Fitting the experimental data of Fig. 2 according to Eq. (4) gives $k_{\text {obs }}$. Because the desorption rates of the thiols are much smaller than their adsorption rates, because of their strong Au-S interactions (i.e., $k_{\mathrm{d}} \ll k_{\mathrm{a}}$ ), we can ignore the term $k_{\mathrm{d}}$. Consequently, $k_{\mathrm{obs}} \approx k_{\mathrm{a}} C$ and, thus, at a constant thiol concentration, $k_{\mathrm{obs}}$ can reflect the magnitude of $k_{\mathrm{a}}$ directly. In addition to singleexponential kinetics, we found that some of the thiols exhibit double-exponential kinetics. In this case, we further modified Eq. (4) to

$F(t)=K_{1}^{\prime}\left[1-\exp \left(-k_{\mathrm{obs} 1} t\right)\right]+K_{2}^{\prime}\left[1-\exp \left(-k_{\mathrm{obs} 2} t\right)\right]$.

Table 3 lists the structures of the different thiols and summarizes their values of $k_{1}$ and $k_{2}$. Please note that, in this paper, 
Table 3

Structures of thiols and their values of $k_{1}$ and $k_{2}$ for the displacement of NRAuNPs at pH $4.0^{\mathrm{a}}$

\begin{tabular}{|c|c|c|c|}
\hline Thiol & Structure & $k_{1}\left(10^{-2} \mathrm{~s}^{-1}\right)$ & $k_{2}\left(10^{-4} \mathrm{~s}^{-1}\right)$ \\
\hline 3-Mercaptopropionic acid (MPA) & $\mathrm{HSCH}_{2} \mathrm{CH}_{2}-\stackrel{\mathrm{O}}{\mathrm{C}}-\mathrm{CH}$ & 1.19 & \\
\hline 11-Mercaptoundecanoic acid (MUA) & $\mathrm{HSCH}_{2}\left(\mathrm{CH}_{2}\right)_{8} \mathrm{CH}_{2}-\mathrm{C}-\mathrm{CH}$ & 0.90 & \\
\hline 16-Mercaptohexadecanoic acid (MHA) & $\mathrm{HSCH}_{2}\left(\mathrm{CH}_{2}\right)_{13} \mathrm{CH}_{2}-\mathrm{C}-\mathrm{CH}$ & 0.55 & \\
\hline Mercaptosuccinic acid (MSA) & $\mathrm{HO}-\mathrm{C}-\mathrm{CH}_{2} \mathrm{CH}-\mathrm{C}-\mathrm{HO}$ & 0.86 & 8.2 \\
\hline meso-2,3-dimercaptosuccinic acid (DMSA) & $\mathrm{HO}-\mathrm{C}-\mathrm{CH}-\mathrm{CH}-\mathrm{C}-\mathrm{HO}$ & 1.46 & 17.7 \\
\hline 2-Mercaptoethanol (2-ME) & $\mathrm{HSCH}_{2} \mathrm{CH}_{2} \mathrm{OH}$ & 2.72 & 19.1 \\
\hline 3-Mercapto-1,2-propanediol (2-MP) & $\mathrm{HSCH}_{2} \stackrel{\mathrm{OH}}{\mathrm{C}} \mathrm{HCH}_{2} \mathrm{OH}$ & 2.83 & 6.5 \\
\hline$N$-(2-Mercaptopropionyl)glycine (MPG) & $\stackrel{\mathrm{CHCH}}{\mathrm{CH}_{3}}-\mathrm{O}$ & 1.46 & 23.4 \\
\hline Glutathione (GSH) & $\mathrm{HO}-\stackrel{\mathrm{O}}{\mathrm{C}}-\stackrel{\mathrm{H}_{2} \mathrm{~N}}{\mathrm{C}} \mathrm{C}\left(\mathrm{H}-\left(\mathrm{CH}_{2}\right)_{2}-\stackrel{\mathrm{C}}{\mathrm{C}}-\mathrm{NH}-\mathrm{HC}-\stackrel{\mathrm{HSCH}}{\mathrm{C}}-\stackrel{\mathrm{O}}{\mathrm{C}}-\mathrm{NHCH}_{2} \mathrm{C}-\mathrm{OH}\right.$ & 0.51 & 6.0 \\
\hline
\end{tabular}

a The concentrations of the thiols and the NRAuNPs are $0.1 \mathrm{mM}$ and $0.3 \mathrm{nM}$, respectively.

all of these values represent $k_{\text {obs. }}$ MPA, MUA, and MHA all have only one value of $k$ (Group 1), while all of the others have two (Group 2). We note that the values of $k_{2}$ are smaller than those for $k_{1}$. There are a number of important factors that affect the rate constants. First, the diffusion rate of the thiols from the double layer to the surface: smaller thiols reach the surface faster. Second, steric effects: it is more difficult for large molecules to gain access to the surface. Third, Coulombic forces between the AuNPs and the thiols: thiols that possess greater negative charge densities experience stronger repulsion from the AuNPs, which are capped with citrate ions, and, thus, we would expect smaller rate constants. The steric effect is evident when considering that the smallest value of $k_{1}$ in Group 1 occurred for MHA, which has the longest chain length [38]. The fluorescence of the NRAuNP solutions in the presence of the following three thiols (at equilibrium) decreased in the order MPA > MUA > MHA. This finding clearly indicates that fewer NR product molecules were displaced from the AuNP surface by the larger-sized thiol molecules. Both 2-ME and 2-MP are neutral molecules and they display rate constants greater than that of MPA, which supports the notion that Coulombic repulsion is an important factor contributing to magnitudes of the rate constants. The fluorescence increases that occurred upon adding 2-ME and 2-MP were much greater than those when adding MUA and MPA; this observation supports the role that steric interactions play in determining the kinetics and thermodynamics of the interactions between thiols and AuNPs. The very small value of $k_{1}$ that we observed for glutathione further supports the roles of both the steric and Coulombic repulsion effects. The fact that the rate constants for DMSA (two thiol groups) are greater than that of MSA (one thiol group) infers that the number of sulfhydryl groups has an affect on the displacement rate. We attribute the slower kinetic processdenoted by the small values of $k_{2}$-as being likely the result of a consolidation of the thiol film formed on the NRAuNP surface [33,38,39]. An imperfect, partial molecular assembly forms at the earlier stage and then it becomes reorganized in a second stage into a more perfectly ordered monolayer [39]. Because of the intermediate consolidation process, the adsorption rate of thiols decreases and thus $k_{2}$ is smaller than $k_{1}$.

Schemes $2 \mathrm{a}$ and $2 \mathrm{~b}$ present cartoons depicting the proposed mechanisms through which the thiols displace the pre-adsorbed NR products. In the cases of MPA, MUA, and MHA, strong repulsion forces exist among the thiol molecules and, therefore, they represented only by values of $k_{1}$, as depicted in Scheme 2a. Unexpectedly, thiols that contain hydroxyl groups (e.g., 2-ME) or amino groups [e.g., GSH ( $\mathrm{p} K_{\mathrm{a} 1}=2.12$ and $\left.\mathrm{p} K_{\mathrm{a} 2}=3.59\right)$ and MPG], or that have flat structures on the AuNP surface in the secondary positions of their main chains (e.g., MSA and DMSA), are characterized by two rate constants $\left(k_{1}\right.$ and $\left.k_{2}\right)$ at $\mathrm{pH}$ 4.0. The proposed mechanism is depicted in Scheme $2 \mathrm{~b}$. With their weaker repulsion and possible formation of hydrogen bonding [40,41] or hydrophobic interactions between thiols, these thiols gain ready access to the surface and, thus, they have an easier time displacing the difficult-to-exchange NR product.

The TEM images in Fig. 3 clearly exhibit that the AuNPs aggregate to different extents once they are bound to the various thiols, which is in good agreement with the red-shift and band broadening of the SPR bands for the NRAuNPs in the presence 


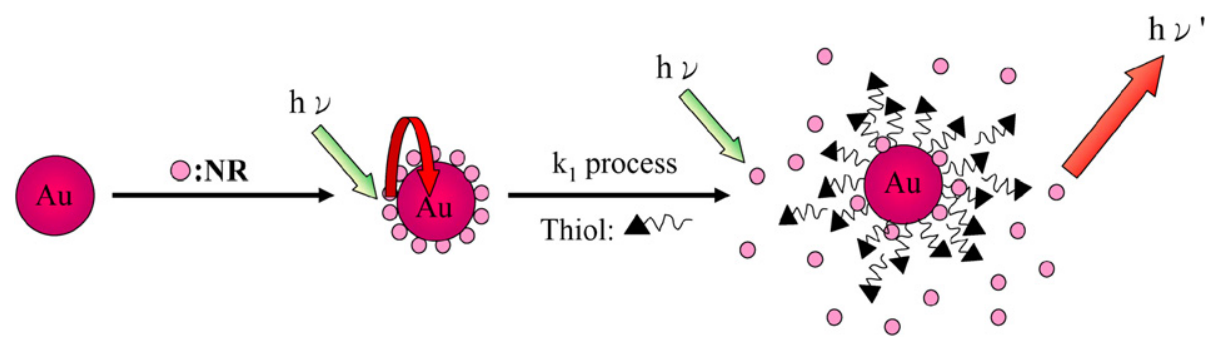

(a)
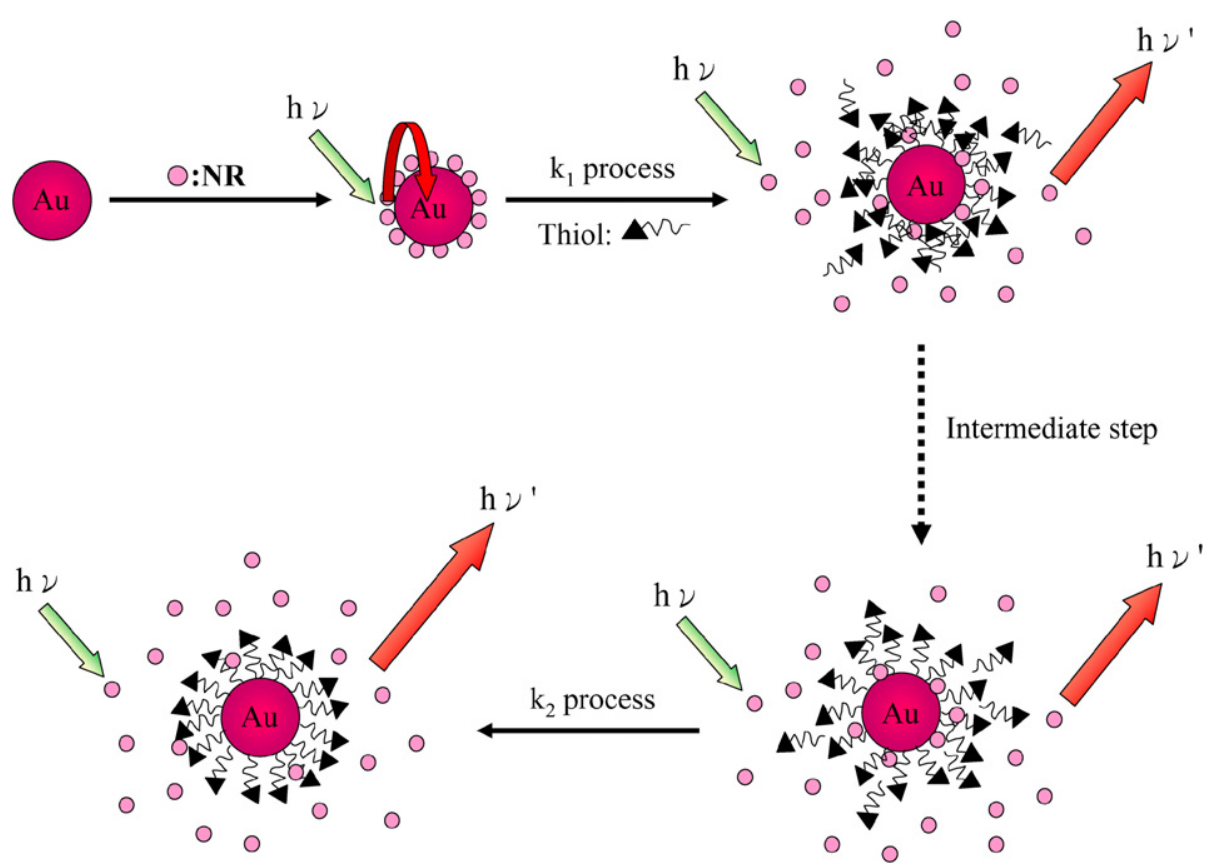

(b)

Scheme 2. A cartoon representation of the displacement by thiols of the NR products adsorbed on AuNPs. Thiols possess (a) $k_{1}$ value; (b) $k_{1}$ and $k_{2}$ values.

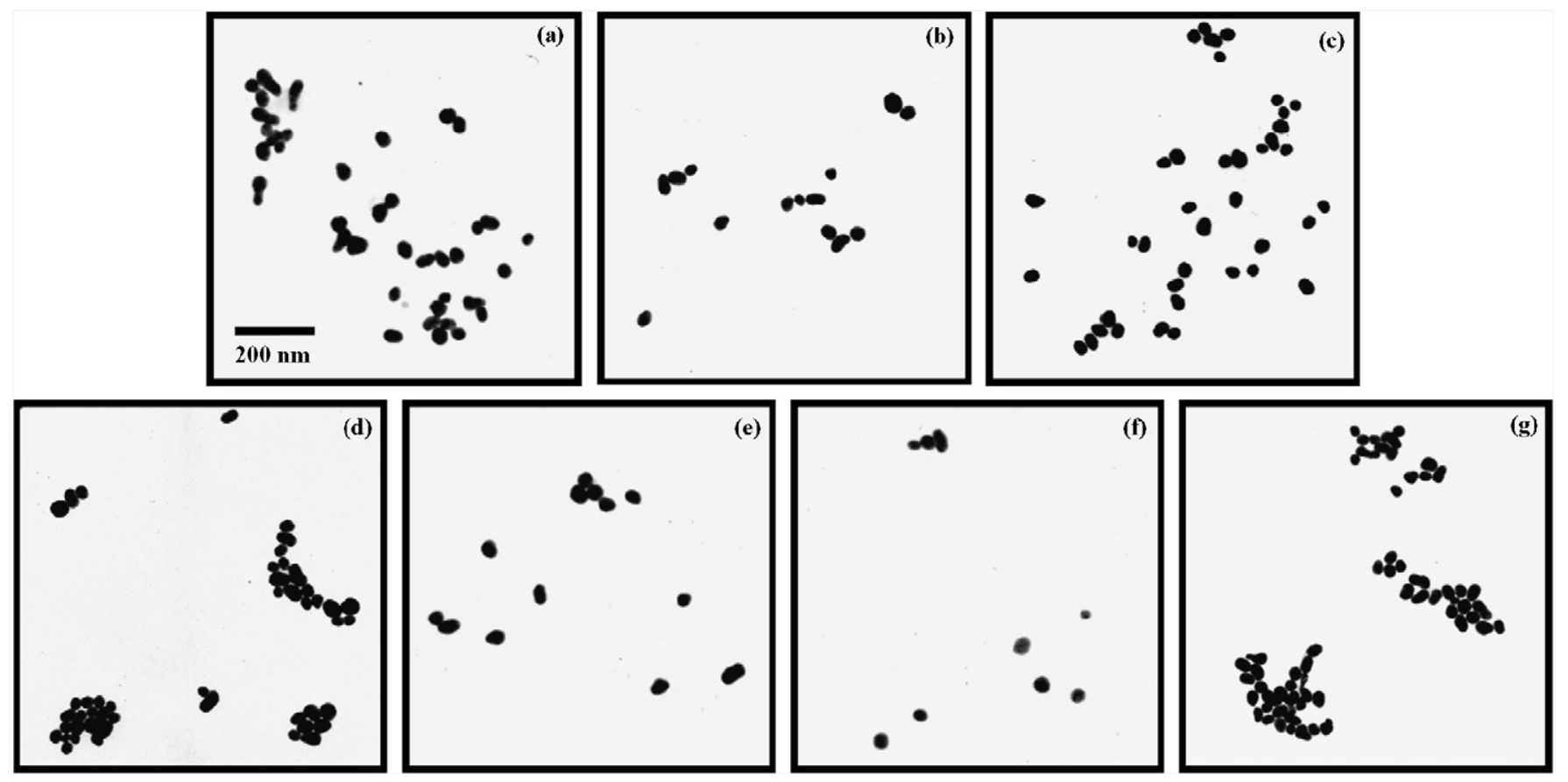

Fig. 3. TEM images of NRAuNPs recorded in the absence and presence of thiols: (a) NRAuNPs, (b) MPG-AuNPs, (c) GSH-AuNPs, (d) MHA-AuNPs, (e) MPA-AuNPs, (f) MSA-AuNPs, (g) DMSA-AuNPs. 
Table 4

Comparison of the kinetic and thermodynamic properties of thiol adsorption using different sizes of AuNPs

\begin{tabular}{|c|c|c|c|}
\hline & \multicolumn{3}{|l|}{ AuNPs (nm) } \\
\hline & 14 & 32 & 56 \\
\hline Concentration of AuNPs (nM) & 3.44 & 0.288 & 0.054 \\
\hline NR saturated adsorption concentration $(\mu \mathrm{M})$ & 1.26 & 0.950 & 0.421 \\
\hline NR adsorption number (NR molecules/particle) & 366 & 3300 & 7836 \\
\hline $\begin{array}{l}\text { Surface coverage } \\
\left(\times 10^{17} \mathrm{NR} \text { molecules } / \mathrm{m}^{2}\right)\end{array}$ & 5.95 & 10.3 & 7.96 \\
\hline Dynamic range $^{\mathrm{a}}(\mathrm{M})$ & $10^{-7}$ to $7 \times 10^{-6}$ & $3 \times 10^{-7}$ to $3 \times 10^{-6}$ & $10^{-7}$ to $10^{-6}$ \\
\hline Regression coefficient $\left(R^{2}\right)^{\mathrm{a}}$ & 0.9963 & 0.9960 & 0.9968 \\
\hline \multirow[t]{2}{*}{$\operatorname{LOD}(\mathrm{nM})^{\mathrm{a}}$} & 20.9 & 8.1 & 19.6 \\
\hline & \multicolumn{3}{|c|}{ Kinetics $\left(k_{1}, \times 10^{-2} \mathrm{~s}^{-1}\right)$} \\
\hline MPG & 2.01 & 1.46 & 1.21 \\
\hline DMSA & 1.78 & 1.46 & 0.561 \\
\hline MPA & 1.57 & 1.19 & 0.373 \\
\hline MUA & 1.31 & 0.90 & 0.553 \\
\hline
\end{tabular}

a Analysis of MPG.

of the thiols. The NRAuNPs are not as stable as the MPGAuNPs, MPA-AuNPs, and GSH-AuNPs at pH 4.0, mainly because of Coulombic repulsion. That is to say, once the small anionic thiols such as MPA and MPG adsorb onto - and the NR product molecules displace from-the surface of the AuNPs, these surfaces become more hydrophilic and possess a greater density of anionic charge, which leads to their greater stability. Although the MHA-AuNPs are stable, their TEM image reveals that they aggregated to a greater extent than the three mentioned above because anionic MHA is relatively hydrophobic when compared with anionic MPA. We also found that the aggregation of the DMSA-AuNPs occurred to a greater extent than that of the MPA and MSA derivatives, possibly because of the stronger interactions that DMSA has with AuNPs (i.e., two $\mathrm{Au}-\mathrm{S}$ bonds per molecule). As a result, the two $\mathrm{COOH}$ groups in DMSA are located relatively close to the gold surface, which leads to relatively weak repulsion forces among the modified AuNPs.

\subsection{Effect of AuNP size}

The size of AuNPs is another important factor in determining the total amounts of adsorbed NR and thiol molecules on each AuNP and the kinetics of thiol displacement. The total number of NR molecules adsorbed on each AuNP should be lower for small-sized AuNPs. In addition, smaller AuNPs have greater curvatures to their structures, which should provide the thiols with more ready access to the surfaces. Thus, we expected the displacement rates for certain thiols to be higher when using small-sized AuNPs. Table 4 lists the saturated NR concentration, the number of adsorbed NR molecules, their surface coverage on each AuNP, and the rate constants; these values are in good agreement with our hypothesis. Table 4 also indicates that the LOD for MPG is $8.1 \mathrm{nM}$ when using the 32nm-diameter NRAuNPs; this value is lower than those obtained when using the 14- and 56-nm-diameter NRAuNPs. The observation of such higher LODs when using smaller NRAuNPs is most likely to be due to fluorescence quenching of the smaller AuNPs that occurs as a result of more collisions-their con- centrations and diffusion coefficients are greater-than those of larger particles. Our reasoning is supported by an increase in the fluorescence intensity (ca. 2.2 fold) observed after centrifugation.

\section{Conclusions}

We have demonstrated the catalytic characteristics of AuNPs through the formation of an NR product $\left(\mathrm{C}_{16} \mathrm{H}_{8} \mathrm{ClNO}_{2}\right)$ from NR $\left(\mathrm{C}_{20} \mathrm{H}_{18} \mathrm{~N}_{2} \mathrm{O}_{2}\right)$ upon the AuNP surface. Because of the greater quantum yield of the NR product, the NRAuNP-based probe is sensitive to the presence of a variety of thiols on the basis of aggregation and fluorescence resonance energy transfer phenomena. We have demonstrated that the degree of aggregation of the AuNPs (i.e., MHA > MPA and DMSA > MSA) depends on the hydrophilicity and structure of the adsorbed thiols. The aggregation of thiol-AuNPs is dependent on several driving forces, including hydrogen bonding, Coulombic, and hydrophobic interactions between the adsorbed thiols. Our study of the kinetics of the displacement of the NR product by thiols suggests that displacement by thiols containing carboxyl residues follows single-exponential kinetics, while those for thiols containing hydroxyl groups or amino groups, or those have flat structures on the AuNP surface, exhibit doubleexponential kinetics.

\section{Acknowledgments}

This work was supported by the National Science Council of Taiwan under contract NSC 95-2113-M-002-026-MY3. We thank Professor Tien-Yau Luh (Department of Chemistry, National Taiwan University) for proposing the mechanism for the formation of NR product.

\section{References}

[1] E. Casero, M. Darder, F. Pariente, E. Lorenzo, J. Martin-Benito, L. Vázquez, Nano Lett. 2 (2002) 577.

[2] K.-Y. Chun, P. Stroeve, Langmuir 18 (2002) 4653. 
[3] S.-Y. Lin, Y.-T. Tsai, C.-C. Chen, C.-M. Lin, C.-H. Chen, J. Phys. Chem. B 108 (2004) 2134.

[4] S.-J. Chen, H.-T. Chang, Anal. Chem. 76 (2004) 3727.

[5] L.M. Demers, C.A. Mirkin, R.C. Mucic, R.A. Reynolds III, R.L. Letsinger, R. Elghanian, G. Viswanadham, Anal. Chem. 72 (2000) 5535.

[6] P. Sandström, M. Boncheva, B. Åkerman, Langmuir 19 (2003) 7537.

[7] M. Aslam, I.S. Mulla, K. Vijayamohanan, Langmuir 17 (2001) 7487.

[8] E.E. Foos, A.W. Snow, M.E. Twigg, M.G. Ancona, Chem. Mater. 14 (2002) 2401.

[9] C.K. Yee, A. Ulman, J.D. Ruiz, A. Parikh, H. White, M. Rafailovich, Langmuir 19 (2003) 9450.

[10] E. Hutter, J.H. Fendler, D. Roy, J. Phys. Chem. B 105 (2001) 11159.

[11] M.M.Y. Chen, A. Katz, Langmuir 18 (2002) 2413.

[12] T. Huang, R.W. Murray, Langmuir 18 (2002) 7077.

[13] K. Busch, J. Piehler, H. Fromm, Biochemistry 39 (2000) 10110.

[14] J.-M.I. Alakoskela, P.K.J. Kinnunen, J. Phys. Chem. B 105 (2001) 11294.

[15] X. Leng, K. Starchev, J. Buffle, Langmuir 18 (2002) 7602.

[16] J.S. Kirk, P.W. Bohn, J. Am. Chem. Soc. 126 (2004) 5920.

[17] E. Dulkeith, A.C. Morteani, T. Niedereichholz, T.A. Klar, J. Feldmann, S.A. Levi, F.C.J.M. van Veggel, D.N. Reinhoudt, M. Möller, D.I. Gittins, Phys. Rev. Lett. 89 (2002), 203002-1.

[18] M. Montalti, L. Prodi, N. Zaccheroni, R. Baxter, G. Teobaldi, F. Zerbetto, Langmuir 19 (2003) 5172.

[19] M.J. Hostetler, A.C. Templeton, R.W. Murray, Langmuir 15 (1999) 3782.

[20] Y.C. Cao, R. Jin, C.A. Mirkin, Science 297 (2002) 1536.

[21] Y.C. Cao, R. Jin, J.-M. Nam, C.S. Thaxton, C.A. Mirkin, J. Am. Chem. Soc. 125 (2003) 14676.
[22] D.S. Karpovich, G.J. Blanchard, Langmuir 10 (1994) 3315.

[23] S. Liao, Y. Shnidman, A. Ulman, J. Am. Chem. Soc. 122 (2000) 3688.

[24] D.B. Hibbert, J.J. Gooding, P. Erokhin, Langmuir 18 (2002) 1770.

[25] G. Frens, Nature 241 (1973) 20.

[26] K.C. Grabar, R.G. Freeman, M.B. Hommer, M.J. Natan, Anal. Chem. 67 (1995) 735.

[27] J.S. Kirk, P.W. Bohn, J. Am. Chem. Soc. 126 (2004) 5920.

[28] M. Haruta, Catal. Today 36 (1997) 153.

[29] M.-C. Daniel, D. Astruc, Chem. Rev. 104 (2004) 293.

[30] N. Ghoneim, Spectrochim. Acta Part A 56 (2000) 1003.

[31] A.K. Dutta, K. Kamada, K. Ohta, J. Photochem. Photobiol. A 93 (1996) 57.

[32] W. Pan, C.J. Durning, N.J. Turro, Langmuir 12 (1996) 4469.

[33] G. Häehner, C. Wöll, M. Buck, M. Grunze, Langmuir 9 (1993) 1955.

[34] C.D. Keating, M.D. Musick, M.H. Keefe, M.J. Natan, J. Chem. Educ. 76 (1999) 949.

[35] Y.-T. Tao, S. Pandiaraju, W.-L. Lin, L.-J. Chen, Langmuir 14 (1998) 145.

[36] Y.-S. Shon, E. Cutler, Langmuir 20 (2004) 6626.

[37] M.R. Kosuri, R. Cone, Q. Li, S.M. Han, B.C. Bunker, T.M. Mayer, Langmuir 20 (2004) 835.

[38] K.A. Peterlinz, R. Georgiadis, Langmuir 12 (1996) 4731.

[39] C.D. Bain, E.B. Troughton, Y.-T. Tao, J. Evall, G.M. Whitesides, R.G. Nuzzo, J. Am. Chem. Soc. 111 (1989) 321.

[40] P.R. Selvakannan, S. Mandal, S. Phadtare, R. Pasricha, M. Sastry, Langmuir 19 (2003) 3545.

[41] S. Mandal, A. Gole, N. Lala, R. Gonnada, V. Ganvir, M. Sastry, Langmuir 17 (2001) 6262. 